\title{
Syntactic Ambiguity Resolution of Cantonese-English Bilinguals: Evidence from Prepositional Phrase and Adverb
}

\author{
Bing Bai \\ China University of Petroleum Beijing, At Karamay, Karamay City, China \\ Email: baibingcup@163.com
}

How to cite this paper: Bai, B. (2018). Syntactic Ambiguity Resolution of Cantonese-English Bilinguals: Evidence from Prepositional Phrase and Adverb. Open Journal of Modern Linguistics, 8, 39-47. https://doi.org/10.4236/ojml.2018.83005

Received: April 2, 2018

Accepted: May 18, 2018

Published: May 22, 2018

Copyright $\odot 2018$ by author and Scientific Research Publishing Inc. This work is licensed under the Creative Commons Attribution International License (CC BY 4.0).

http://creativecommons.org/licenses/by/4.0/

\section{(c) (i) Open Access}

\begin{abstract}
Syntactic ambiguity resolution is influenced by multiple constraints. A sentence-picture matching task tested the attachment of prepositional phrase (PP) such as on the chair within an utterance like Mary ate the apple on the chair. PP attaching to NP2 (the apple) is called low attachment (LA), while PP attaching to $\mathrm{V}$ (ate) is called high attachment (HA). A comprehension task tested the attachment of adverb such as quickly within an utterance like the story Mary was reading quickly put her little sister into sleep. The checked adverbs attaching to the verb of the embedded clause (VEC) and to the verb of the main clause (VMC) were analyzed. Cantonese-English bilinguals were grouped according to their L2 proficiency. Results reflected that 1) both groups showed preference to HA for PP, 2) LP group showed preference to VMC for adverb attachment; 3 ) there is a large gap in LA between LP and HP, suggesting that syntactic ambiguity resolution involves interaction of all the information available. The relationship between language proficiency and parsing is not so definitely supported by the results.
\end{abstract}

\section{Keywords}

Syntactic Ambiguity, Cantonese-English Bilinguals, Parsing, Language

Processing

\section{Introduction and Literature Review}

Syntactically ambiguous structures can be utilized to test parsing strategies. When it comes to language processing, two kinds of models are put forward. More specifically, models like garden-path model (Frazier \& Fodor, 1978) claim that learners merely resort to pure syntactic information in syntactic ambiguity 
resolution, at least at initial processing stage, and that semantic and discourse information only impact syntactic ambiguity at a later stage. However, language learning might involve cognitive activities. Compared with models highlighting purely syntactic information, interactionist model favored by MacDonald (1997) assumes that learners rely on all the information available, including syntactic information (Branigan, Pickering, \& McLean, 2005; Clifton, Speer et al., 1991), lexical information (Frenck-Mestre et al. 1997), and semantic information (Boland \& Blodgett, 2006; Carson \& Gibson, 1999). It is worth noting that things become more complicated in $\mathrm{L} 2$ disambiguation because of a wide range of factors, namely L2 exposure, possible transfer of parsing mechanism from L1 to L, syntactic information such as argument structure, lexical information and referential context.

Whether L2 exposure can impact parsing strategy is showed by both on-line and off-line experiments. Dussias et al. $(2003 ; 2007)$ claimed that there is a remarkable difference among Spanish-English bilinguals in their preference of relative clause (RC) attachment, namely, the temporarily ambiguous NP such as the sister of the actor, because they were exposed to L2 at different level. While there was no conclusive argumentation, both the Construal and the Tuning hypothesis attempt to explain the attachment preference based on discourses with language-specific grammatical information and language input respectively. Specifically, low attachment (LA) frees speakers from cognitive load as in Late Closure hypothesis, which was revealed by the investigation of PP such as Brutus hit the gladiator with the shield with his bare hands attachment (Frenck-Mestre et al., 1997). They suggested that bilinguals perform a similar syntactic parsing strategy in L1 and L2, and that bilinguals are more likely to attach the incoming information with the most recently processed constituents. Therefore, whether there truly exists an overriding syntactic impact in spite of apparent effect of lexical cues on parsing. Also, it is under debate whether bilinguals employ the same parsing strategies in L2 and what constraints influence L2 parsing on-line.

The parsing strategies, such as the Late Closure, underscore the overriding importance of syntactic information including argument structure. A relationship between production and comprehension of PPs is revealed by Branigan, Pickering, \& McLean (2005), who discovered that participants are more likely to produce HA in the description-sentence matching task. Contrary to the results above, Carson and Gibson (1999) found that reading time of noun argument is faster than verb attachment and they suggest a recency-driven parsing strategy, similar to Minimal Attachment. Time-saving parsing strategy cannot fully fix ambiguity resolution of without awareness of the lexicon features. Clifton et al. (1991) proposed that processing difficulty exists in attaching PP as the modifier of verbs instead of noun argument, suggesting that all lexical information is used in syntactic ambiguity resolution. By manipulating lexical information and argument structure of the verb in the reduced relative clause (RC), Juffs (1998) found that learners of different language background show differences in sentence processing. Chinese speakers were slower in terms of reading time in the disam- 
biguating region (i.e. the main verb), and there was a significant difference between native speakers and L2 speakers in processing a sentence consisting purely intransitive verbs. The processing difficulty of PP lies in the debatable boundary between argument and modifying adjunct. A distinction is found between adjunct and argument matters in temporarily syntactic sentences containing a complex PP, because they are subject to different attachment mechanism according to the Argument Structure Hypothesis (ASH). As demonstrated by Boland and Blodgett (2006), argument attachment is easier than adjunct attachment, meaning that argument attachment is lexically guided while adjunct attachment is not. Apart from the complex PP attachment, the relatively flexible word order of adverbs is investigated to shed light on possible factors influencing ambiguity resolution. However, this does not suggest the position of adverbs is the source of interpretation. Instead, the source lies in the syntactic property of adverbs (Ernst. 2002). Concerning the relatively flexible position, adverb can cause syntactic ambiguity like the following utterance the dog Marry keeps carelessly broke the glasses, in which carelessly can be attached to keeps or broke, yielding two different readings. This study investigates the Chinese-English bilinguals' attachment preference for adverbs and PPs expressing manners, frequency and mental-attitude.

Either garden-path model or the Late Closure parsing strategy is derived from the Sausage Machine, whose central claim is that syntactic ambiguities are resolved by all the available syntactic information. However, the constraint-based model predicts that language users can select a particular interpretation of an ambiguity basing on lexical information. In favor of the constraints-based model, Spivey and Tanehaus (1998) shed light on the importance of the multiple constraints in resolving reduced RC ambiguity, including 1) the semantics fitting between the thematic role of the higher NP in main clause and the reduced RC; 2) the lexical frequency of serving as a past participle or past tense; 3 ) the feature of transitivity or intransitity; and 4) the following information. These findings suggest that purely syntactic information cannot fully explain L2 parsing and that sentence parsing strategy can be language specific. An off-line grammaticality judgment task of RC following a complex noun phrase like the secretary of the professors revealed that lexical cues counts much in L2 parsing Felser et al. (2003).

Apart from linguistic constraints, working memory also affects syntactic parsing and is more remarkable for bilinguals. Hoppa (2014) reported the effects of working memory on L2 RC attachment preferences in German-English bilinguals, and the result showed that high proficient L2 learners show parsing patterns similar to those of native counterparts.

\section{Research Questions and Methods}

This study investigates 1) whether Chinese-English bilinguals resort to pure syntactic information or the interaction of all the information available in the two checked structures; 2) whether participants have a preference for the attachment; and 3) whether L2 proficiency has a significant influence on compre- 
hending ambiguous structures. The study begins with a general review of syntactic ambiguity resolution followed by two priming tasks: experiment 1 for PP attachment and experiment 2 for adverb attachment.

\subsection{Experiment 1}

\section{Participants}

10 Cantonese-English bilinguals aged between 33 and 37, all of whom have no speech or listening loss and are grouped to high proficiency group (HP). HP is defined by the fact that they either studied in UK/US for 10 years or scored Grade $\mathrm{C}$ or above in HKALE English (Hong Kong Advanced Level Examination) and use English daily, while LP is defined by Grade E or F in HKALE English with limited use of English.

\section{Items}

This experiment is a sentence-picture matching task to investigate whether how the participants interpret ambiguous sentences. There are target 12 stimuli and 6 fillers. All the stimuli are of the same structure of NP1 +V +NP2 + PP (illustrated by (a) below). The verb used in the tested items are found, saw, made, walked, ate, drew, bought, hit, watched, played, sings, used, wrote, poked, chased, talked. All the stimuli are coupled with three pictures. Specifically, regarding target stimulus, two pictures corresponds to two possible readings and one impossible reading. Also, each filler are coupled with two impossible readings and one correct reading. The tested items are listed in the appendix (please refer to https://www.dropbox.com/s/vlk8wk78zlqzv1w/Appendix.pdf?dl=0).

(a) $N P 1+V+N P 2+P P$

The spy saw the police with binoculars.
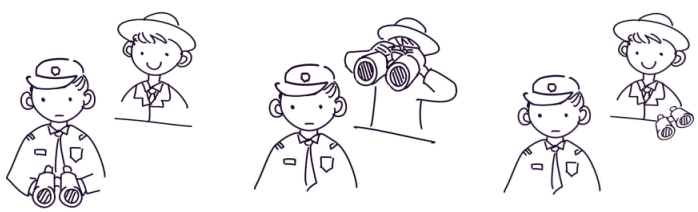

\section{Procedures}

The experiment, whose target stimuli and fillers are randomized in order, is conducted in the context of no distraction of noise. Not informed the aim of the experiment before the task is finished, they were asked to listen to a recording of the stimuli (which are recorded by a native speaker and proofed by another to ensure no prominence existing in prosody or pause) followed by the pictures, and then were asked to identify the picture corresponding to their interpretations.

\section{Results \& Data Analysis}

Answers of all the participants are clipped in appendix (please refer to https://www.dropbox.com/s/vlk8wk78zlqzvlw/Appendix.pdf?dl=0). Their answers were analyzed as Table 1.

The average error rate of targeted questions of HP group is $1.67 \%$ and that of LP group is $3.33 \%$. Mean Preferred is the average rate of preferred options which 
Table 1. Proportion of LA \& HA in Experiment 1.

\begin{tabular}{cccc}
\hline & & High Proficiency & Low Proficiency \\
\hline \multirow{2}{*}{ LA } & Mean Preferred & $8.33 \%$ & $26.67 \%$ \\
& Mean Total & $25.00 \%$ & $35.00 \%$ \\
HA & Mean Preferred & $90.00 \%$ & $71.67 \%$ \\
& Mean Total & $90.00 \%$ & $78.33 \%$ \\
\hline
\end{tabular}

participants chose, while Mean Total is the average rate of chosen options of that attachment. The figures show that both groups preferred pictures representing HA structures. $90 \%$ of the answers of HP group are pictures of HA, more than that of LP group. On the other hand, few participants of HP group chose pictures of LA than that of LP group.

Additionally, it is noted that Participant 2 of HP group has a higher error rate than the others. He was questioned about the errors and it turned out that his loose criteria of determining acceptable options led to the errors. For example, in question 2, he argued that the binoculars were located too close to the spy that he thought the spy had the possession of the binoculars. And in question 8 , he argued that $\$ 20$ dollars could also buy the newspaper and Mary did not keep the change. Since most of his errors are in the filler questions, it does not affect our analysis in general. Participant 4 of LP group was also questioned about his errors. He said it was due to the fact that the recordings of the five questions were too fast, but he did understand the others. Again, most of his errors are in the filler questions and it does not affect our analysis in general.

\section{General Discussion}

Considering the parsing strategy, a preference for LA was predicted because PP is structurally adjacent to NP2. Whether this is due to working memory capacity or efficiency-driven model should be further discussed. Theoretically, as O'Grady (2008) proposed, sentence processing has a general computational feature: a propensity to operate on pairs of elements. In other words, the processing operates in a linear order for efficiency. If sentence parsing is substantially influenced by information processing, people are more likely to attach an ambiguous phrase to the currently processed phrase to construct a simple allowable structure. For example, after the fulfillment of operation of NP Mary and V ate the apple in the utterance Mary ate the apple on the chair, PP on the chair is attached to the most currently processed NP the apple. Therefore, PP, hopefully, will be attached to the most adjacent NP the apple because there is no intervened overt node between the two. Generally speaking, the result is not a proponent for the efficiency-driven strategy. A possible explanation may attribute to the lexicon representation and the mixed boundary between argument and adjunct. Arguments and their head are called primary phrases, and their attachment must rely on structure principles such as Minimal Attachment, but adjuncts are associated with lexicon-specific information and real world knowledge as sug- 
gested by Frazier and Clifton (1996). They explicitly claimed that PPs in V-NP-PP constructions are initially minimally attached to the VP. This explains the reason why participants chose picture-description corresponding to LA reading. A pure syntactic reliance cannot fully explain syntactic resolution without association to lexically stored information and contextual knowledge.

Bilinguals are advantageous over their monolingual peers concerning disambiguating capacity, and this is also supported by this off-line experiment. We thus predicted that the inhibitory control ability at low proficiency level would outperform that at high proficiency level, provided that the PP structure is allowable (i.e. LA). The rationale behind the prediction is that 1) conceptual and lexical information is used to avoid costly analysis; 2) the less differentiated boundary between adjunct and argument structure give rise to processing preference. However, this is only partially born out and supported by the performance that there is a large gap in LA between LP (26.67\%) and HP (8.33\%). We found that 1) either HP or LP group shown low preference for LA, meaning that language proficiency has marginal effect parsing structure and that participants are not sensitive to recency in processing. Compared with the superiorly syntactic requirement for argument, it is not necessarily possible to put adjunct into optional or even irrelevant information in processing for participants of low working memory. Concerning the variant performance we observed, we apply the explanation given by Gass \& Lee (2011) that working memory is stable, but one's ability to fully utilize it varies with language proficiency. The relationship between language proficiency and parsing is not so definitely supported by the results.

\subsection{Experiment 2}

\section{Participants}

Participants in experiment 1 also participated in experiment 2.

Items

This experiment is a comprehension task to investigate whether the tested adverb is attached to the verb of the embedded clause (VEC) or the verb of main clause (VMC). 12 target stimuli involving by syntactic ambiguity and 6 fillers without ambiguity are constructed. All the stimuli are of the same structure of $\mathrm{NP}+\mathrm{RC}+$ adverb + VP. The checked adverbs express manners, frequency and mental-attitude. All the stimuli couple with three simple sentences. Specifically, each target stimulus are coupled with two possible readings and one fake reading. Each filler are coupled with two fake reading and one correct reading. The checked adverbs express manners, frequency and mental-attitude. All the stimuli couple with three simple sentences, as illustrated by the sample in 1). The items are listed in the appendix (please refer to https://www.dropbox.com/s/vlk8wk78zlqzv1w/Appendix.pdf?dl=0).

1) The director who attends the meeting frequently amends the agenda.

a). The director attends the meeting frequently. 
b). The director amends the agenda frequently.

c). The meeting amends the agenda frequently.

\section{Procedures}

The target stimuli and fillers are randomized in order. The environment for experiment is the same as that of experiment 1 . Participants were told merely in advance that they needed to complete the task. Participants were asked to read the stimuli silently and choose the answer(s) according to their understanding.

\section{Results \& Data Analysis}

Answers of all the participants are clipped in appendix (please refer to https://www.dropbox.com/s/vlk8wk78zlqzv1w/Appendix.pdf?dl=0). Their answers were analyzed as Table 2 below.

The average error rate of targeted questions of HP group is $10.00 \%$ and that of LP group is $21.67 \%$, which are both higher than that in experiment 1 . Mean Preferred is the average rate of preferred options which participants chose while Mean Total is the average rate of chosen options of that structure. Sentences of this experiment involve embedded clause, which increases the difficulties in resolution. Participants of HP group successfully identified the ambiguity for 4 questions in average, which is slightly higher than participants of LP group (3 questions in average). The reason may be the fact that this is an off-line task which participants were allowed to re-read the questions and options more times. There is no preference on VMC/VEC structures in general for HP group, while LP group tends to choose options of VMC structures as their answers.

\section{Discussion}

It is predicted that there would be preference for the adverb attachment to VMC due to processing load. Results generally supported the prediction. The first possible explanation may go to the universal parsing mechanism. For example, the adverb frequently in the utterance the director who attends the meeting frequently amends the agenda is intervened by the NP the meeting, and therefore it is costly to attach the adverb to the verb of embedded clause, suggesting that efficiency-driven mental parsing mechanism works more remarkably at LP. Late Closure in essence is a principle about locality, and the performance of either HP or LP suggests that 1) bilinguals are not strictly subjected to the universal locality principle in term of adjunct attachment; and 2) proficiency in L2 plays fewer roles in disambiguation.

Table 2. Proportion of VMC \& VEC in Experiment 2.

\begin{tabular}{cccc}
\hline & & High Proficiency & Low Proficiency \\
\hline \multirow{2}{*}{ VMC } & Mean Preferred & $46.67 \%$ & $56.67 \%$ \\
& Mean Total & $63.33 \%$ & $71.67 \%$ \\
\multirow{2}{*}{ VEC } & Mean Preferred & $46.67 \%$ & $33.33 \%$ \\
& Mean Total & $63.33 \%$ & $43.33 \%$
\end{tabular}


Another explanation goes to the lexical information stored in the adverb. Similar to the explanation of experiment 1 about the PP attachment, we hereby apply the proposal of the non-primary phrases (Frazier \& Clifton, 1996) consisting non-obligatory constituents to the results. The adverb can be non-primary phrase of main clause or the embedded clause, but it was not construed or associated to the current processed constituents and interpretation based on all the information available. For the participants, they might place less reliance on the word order (i.e. either the adverb is prior to or following the verb of the main clause). Instead, they focused on the probabilities of the event, whereas more possible and allowable according to their knowledge. This may explain why participants of HP exhibited no preference for VMC or VRC. In other words, the processing of non-primary phrases relies on a set of information including contextual and world knowledge but not structural information. This is a proponent of determinants of adjunction site claimed by Ernst (2002) that adjunction site is primarily determined by semantically motivation and lexically specified selection needs, which refers to events, prepositions, time and predicates.

\section{Conclusions}

How language learners process language in real time reveals how they comprehend the constructions in a language. Interface between syntax and other language domain sheds light on the mystery of human mind.

In general, this study revealed that Chinese-English bilinguals mostly rely on all the information available rather than pure syntactic information in syntactic ambiguity resolution. However, our study did not show significant relationship between language proficiency and parsing.

The results may suggest 1) that syntactic information and lexical stored information carry more weight than other domains in ambiguity resolution because of the inborn knowledge of phrase hierarchy; 2) that the cognitive advantage of bilingual (i.e. inhibitory control ability) is good to ambiguity resolution; and 3) that either interpretation of the two possible interpretation reveals the prominence of linguistic information. This study not only benefits those who conduct a cross-cultural communication or teaching Chinese as second language, but suggests syntactic information interfaces with semantic meaning alike in comprehension of ambiguous sentences.

\section{Limitations}

Language contact is an independent variable exerting effect on the results. Although participants involved in this study are Cantonese-English bilinguals, they have exposure to Mandarin Chinese or other languages. Besides, the stimuli used in this study were practical but controversial sometimes. For example, stimulus 14 in experiment 1 and stimuli $6 \& 10$ in experiment 2 are misleading, and we acknowledged this issue. Finally, further research might deepen the effects of lexicon information on ambiguity resolution, in that two verbs (i.e. chased, talked) used in experiment 1 are intransitive while others are transitive. 


\section{Acknowledgements}

Miss. NG Yinying collected data for this study in HK and drew the pictures.

\section{References}

Boland, J. E., \& Blodgett, A. (2006). Argument Status and PP-Attachment. Journal of Psycholinguistic Research, 35, 385-403. https://doi.org/10.1007/s10936-006-9021-Z

Branigan, H. P., Pickering, M. J., \& McLean, J. F. (2005). Priming Prepositional-Phrase Attachment during Comprehension. Journal of Experimental Psychology Learning Memory and Cognition, 31, 468-81. https://doi.org/10.1037/0278-7393.31.3.468

Carson, T., \& Gibson, E. (1999). Prepositional Phrase Attachment. Journal of Memory and Language, 40, 409-431.

Clifton, C., Speer, S., \& Abney, S. P. (1991). Parsing Arguments: Phrase Structure and Argument Structure as Determinants of Initial Parsing Decisions. Journal of Memory and Language, 30, 251-271. https://doi.org/10.1016/0749-596X(91)90006-6

Dussias, P. E. (2003). Syntactic Ambiguity Resolution in L2 Learners: Some Effects of Bilinguality on L1 and L2 Processing Strategies. Studies in Second Language Acquisition, 25, 529-557. https://doi.org/10.1017/S0272263103000238

Dussias, P. E., \& Sagarra, N. (2007). The Effect of Language Exposure on Syntactic Parsing. Bilingualism: Language and Cognition, 10, 101-116. https://doi.org/10.1017/S1366728906002847

Felser, C., Roberts, L., Gross, R., \& Marinis, T. (2003). The Processing of Ambiguous Sentences by First and Second Language Learners of English. Applied Psycholinguistics, 24, 453-89. https://doi.org/10.1017/S0142716403000237

Frazier, L., \& Clifton Jr., C. (1996). Construal. Cambridge, MA: MIT Press.

Frazier, L., \& Fodor, J. D. (1978). The Sausage Machine: A New Two-Stage Parsing Model. Cognition, 6, 291-325. https://doi.org/10.1016/0010-0277(78)90002-1

Frenck-Mestre, C., \& Pynte, J. (1997). Syntactic Ambiguity Resolution While Reading in Second and Native Languages. Quarterly Journal of Experimental Psychology, 50A, 119-148. https://doi.org/10.1080/027249897392251

Gass, S. M., \& Lee, J. (2011). Working Memory Capacity, inhibitory Control, and Proficiency in a Second Language. Studies in Bilingualism, 43, 59-84.

https://doi.org/10.1075/sibil.43.06gas

Hoppa, H. (2014). Working Memory Effects in the L2 Processing of Ambiguous Relative Clauses. Language Acquisition, 21, 250-278.

https://doi.org/10.1080/10489223.2014.892943

Juffs, A. (1998). Main verb vs. Reduced Relative Clause Ambiguity Resolution in L2 Sentence Processing. Language Learning, 48, 107-147.

https://doi.org/10.1111/1467-9922.00034

O'Grady, W. (2008). Innateness, Universal Grammar, and Emergentism. Lingua, 118, 620-631. https://doi.org/10.1016/j.lingua.2007.03.005

Spivey, M. J., \& Tanenhaus, M. K. (1998). Syntactic Ambiguity Resolution in Discourse: Modeling the Effects of Referential Context and Lexical Frequency. Journal of Experimental Psychology: Learning Memory Cognition, 24, 1521-43.

https://doi.org/10.1037/0278-7393.24.6.1521 